\section{NASA \\ Technical \\ Paper \\ 2629}

August 1986

\title{
Pulse Code Modulation (PCM) \\ Data Storage and Analysis \\ Using a Microcomputer
}

David E. Massey

$$
\begin{aligned}
& \text { (NASA-TP-2624) EULSE CODE MCLULAIION (PCM) } \\
& \text { DATA STOEAGE ANL ANALYSIS USING A } \\
& \text { MICECCOMEUTEA (NASA) \& P }
\end{aligned}
$$

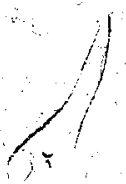


NASA

Technical

Paper

2629

1986

Pulse Code Modulation (PCM)

Data Storage and Analysis

Using a Microcomputer

David E. Massey

Goddard Space Flight Center

Wallops Flight Facility

Wallops Island, Virginia

National Aeronautics

and Space Administration 
PULSE CODE MODULATION (PCM) DATA

STORAGE AND ANALYSIS USING A MICROOOMPUTER

\author{
David E. Massey \\ NASA Goddard Space Flight Center \\ Wallops Flight Facility \\ Wallops Island, VA 23337
}

\title{
INTRODUCTION
}

Within the last few years, as Sounding Rocket Program activity increased, more and more PCM encoders had to be configured and programmed to transmit data from these rocket payloads. The configuration and format are unique to the vehicle and are rarely the same. The encoder is configured by programing an EPROM memory with the appropriate word and frame structure to accormodate all the science and housekeeping data channels. Figure 1

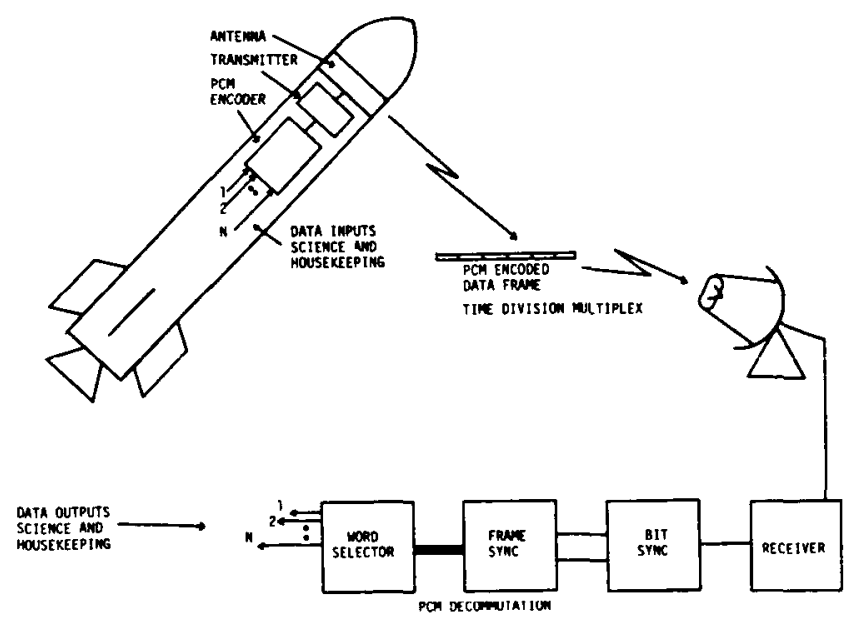

Figure 1.-Sounding rocket PCM data link shows a typical way data is transmitted to the ground using PCM. As would be expected, when output increases, so do the problems due to the everchanging PCM encoder configurations and format programing. The method used for years at Wallops Flight Facility to examine these PCM encoders has been to utilize a standard PCM decommutator, word selector, and strip chart, which do the job but are time-consuming. Clearly, a better, faster, more intelligent method of examining the encoders was needed. This device would be capable of decommutating and storing PCM telemetry data frames for display and analysis, helping to speed up configuration.

The current widespread use of microcomputers has led to the creation of very low-cost instrumentation. A PCM storage device/data analyzer was designed and built for use on the NASA Sounding Rocket Program for PCM encoder configuration and testing. This storage device/ 
data analyzer is a peripheral plug-in board especially constructed to enable a personal computer to store and analyze data from a PCM source. This board and custom-written software turn the computer into a snapshot PCM decommutator which will accept and store many hundreds or thousands of PCM telemetry data frames, then sift through them repeatedly. These data can be converted to any number base and displayed; examined for any bit dropouts or changes -- in particular, words or frames; graphically plotted; or statistically analyzed. These methods help to solve encoder problems.

\section{PROBLEM SOLVING}

Channel Cross Talk.-One of the most evasive problems encountered in PCM encoders is channel cross talk. For example, when stimulating a particular channel or channels on an encoder (see Figure 2), an adjacent channel will pro-

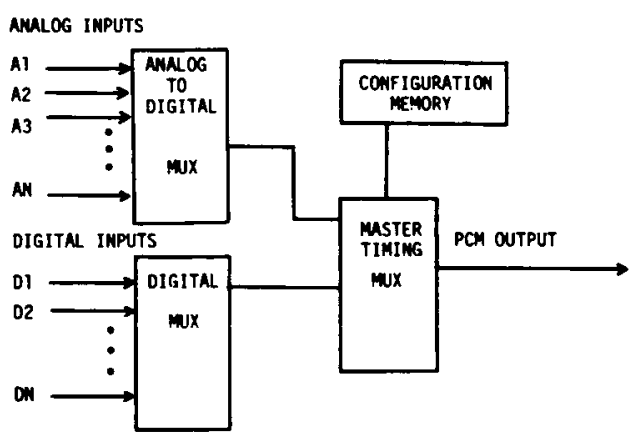

Figure 2.-PCM encoder duce a bit or two intermittently or actually follow a signal that it was not programmed to pick up. Experience has shown that the storage device will work well in this problem area, if you take the cross talk channel as a stop trigger, which enables the storage device to freeze the thousand or so frames previous to the occurrence of the cross talk. This allows examination of other channels prior to the event in question.

Renegade Encoder.-This is an encoder which is outputting PCM data but will not hold solid frame sync lock on a decommutator. The storage device will take in these frames and display them, the operator can sift through this data to locate the partial frame sync pattern, or determine that there is no pattern which in turn should lead to correcting the problem.

Commutation.-Super- or sub-commutated words can easily be evaluated for correct positioning within the frame. Bit glitches and unknown changes can be seen using the graph plot mode by stimulating the channel in question with a known signal; this can be displayed on a graph. If any bit anomalies occur, they will be seen on this picture. A typical lowfrequency signal with two-bit glitches is shown in Figure 3.

The PCM storage device can, of course, be 


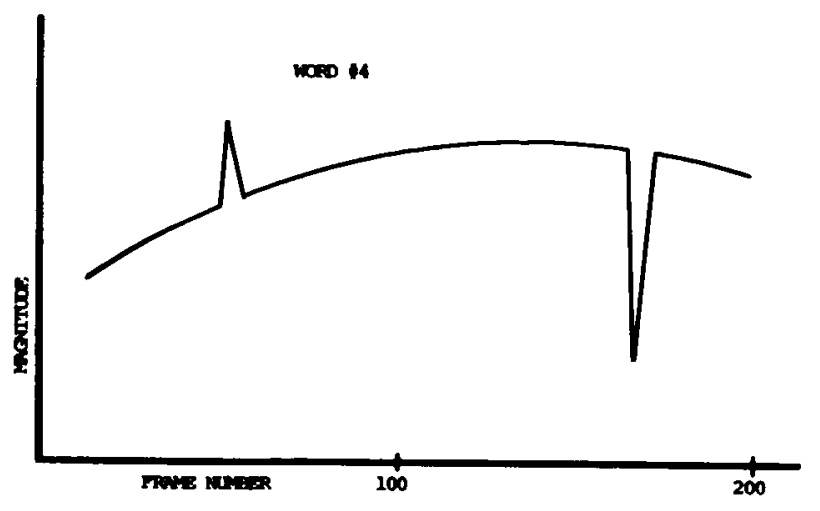

Figure 3.-Graph mode plot of word showing bit glitches

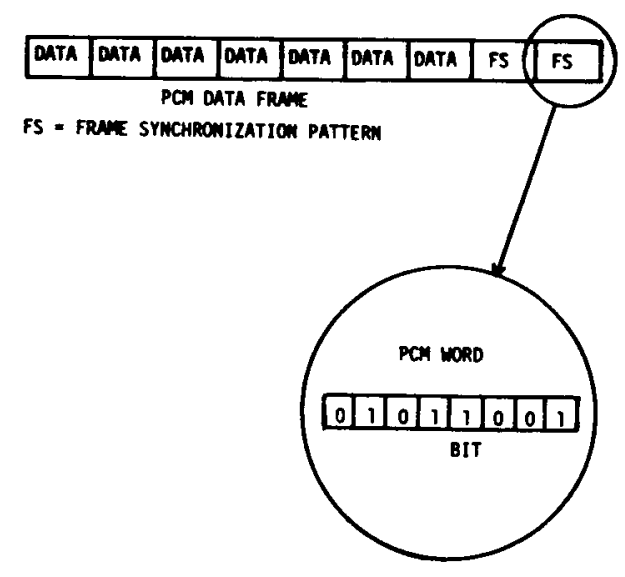

Figure 4.-PCM frame

used to verify correct number of words in a frame, correct word position (see Figure 4), and correct data in a particular subframe. By examining stored frames in a word comparison mode, incorrect data can be found. A typical frame displayed on screen with decimal value conversions is shown in Table I.
TABLE I.-PCM FRAME DISPLAY

$$
\text { PCM FRAME \# } 1
$$

WORD DATA

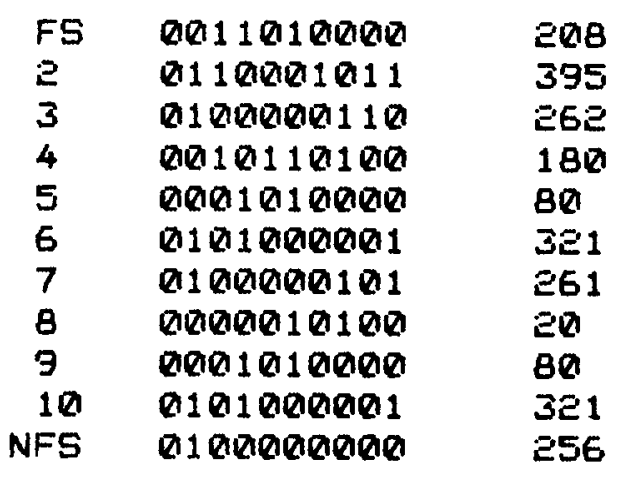

HARDWARE

The hardware of this device is remarkably simple, consisting mainly of a plug-in peripheral board (see Figure 5). The board contains 16 kilobytes of static RAM, address counter/decoder, byte counter, control logic, shift register, and PIA interface port. The 16-kilobyte RAM has 131,072 bits in it, which is the present capacity of PCM storage. The PCM

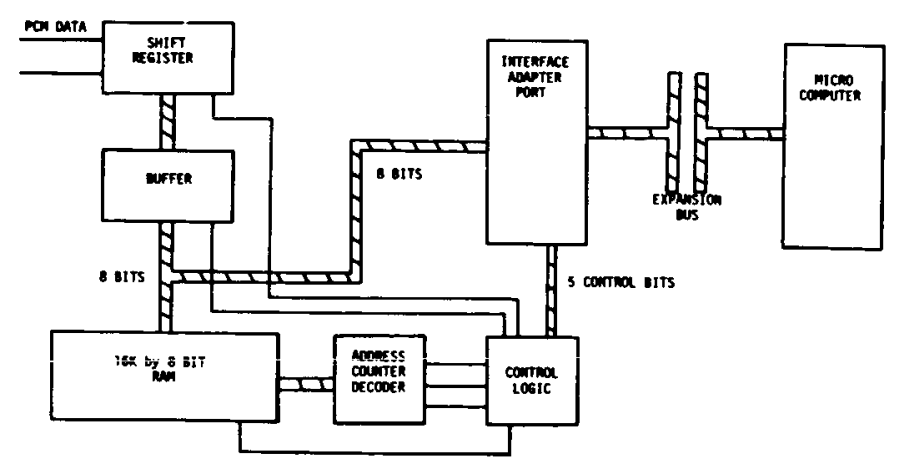

Figure 5.-Hardware block diagram of plug-in board 
data enters the shift register to be converted to 8-bit-wide data. (This has nothing to do with the size of the PCM word in the format.) After it is converted, then it is stored in RAM. Once started into operation, this is a windowing device; i.e., the latest PCM data is always being stored in RAM. When the device is given a stop trigger, either manually or automatically, the last 131,072 bits that were transmitted are frozen. The present board has been tested to five million bits per second (MBPS) with no anomalies. After storage of data, a particular word and scanning all other the microcomputer starts to load bits from the storage device RAM into main computer RAM and begins to process these bits according to the appropriate software algorithm.

\section{SOFTWARE}

As with most microcomputer instruments (any computer application for that matter), software is the key to a good system. In keeping with this thought, the storage device has some long, fairly complex software written for its operations. The software operates in three main modes: frame display/scan, graph plot, and word compare (see Table II). The frame display/scan mode offers screen display of a frame of data (as in Table I) grouped into words and numbered. This data can be examined further by selecting
TABLE II.-SOFTWARE COMMAND MENU

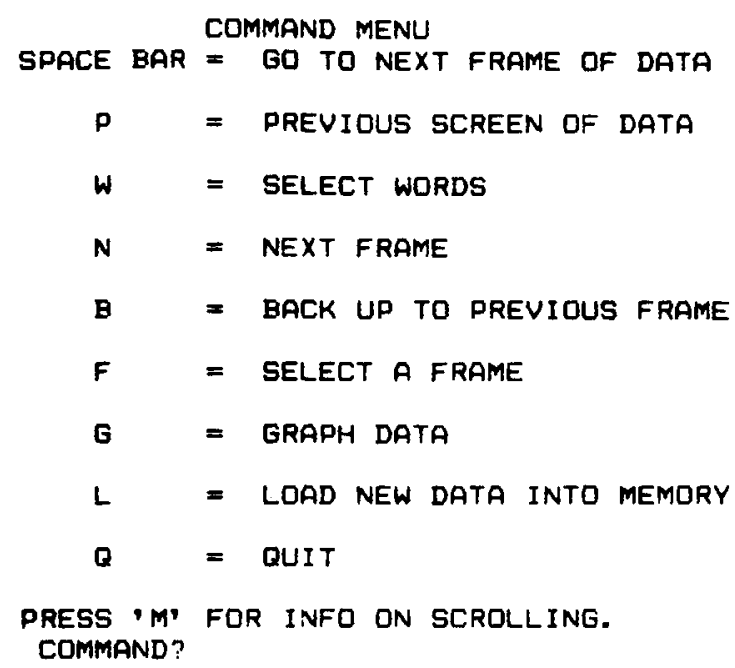
frames for this word. This mode can also select and examine frames of data in sequence and at random. The graph plot mode is a very valuable tool. It allows the selection of a particular word in a PCM frame and plots this word in every stored frame on a graph. This is effectively a graphical digital-to-analog converter. The last mode is the word compare mode; this allows comparison of a word or words in all stored frames. These comparisons can be for changes in data values or other statistical functions.

\section{EXPANSION}

Obviously, 131,072 bits of PCM storage are not enough when dealing with PCM systems approaching one MBPS. A new board containing two to eight million bits is being designed using the new 
252K dynamic RAM chips, thus raising the complexity of the hardware slightly. The only changes in software are to increase pointer addresses to be able to read all storage RAM. Using the 256K dynamic RAM and a LSI controller, the capability of eight million bits exists on one board. This would represent a nice amount of storage, say 10 seconds of data at $800 \mathrm{~K}$ bits/second or about 50,000 frames of average size.

SUMMARY

This instrument does not try to do it all; it is no match for a real-time telemetry data processing computer (which costs hundreds of thousands of dollars). It does fill a void for which no suitable replacement could be found for such a low cost.

NASA Goddard Space Flight Center

Wallops Flight Facility

Wallops Island, VA 23337

August 1985 


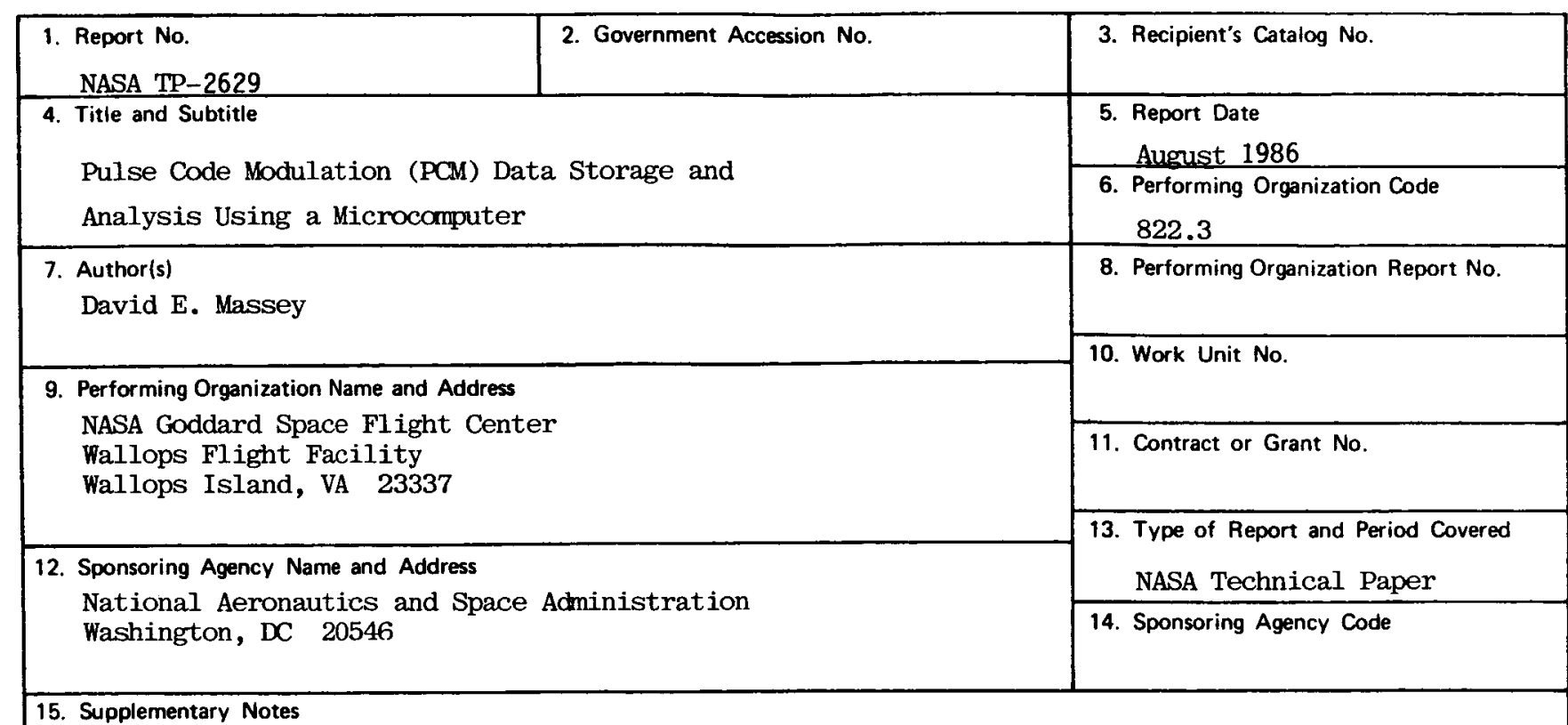

16. Abstract

The current widespread use of microcomputers has led to the creation of some very low-cost instrumentation. A PCM storage device/data analyzer -- a peripheral plug-in board especially constructed to enable a personal computer to store and analyze data from a PCM source -- was designed and built for use on the NASA Sounding Rocket Program for PCM encoder configuration and testing. This board and custam-written software turns a computer into a snapshot PCM decommutator which will accept and store many hundreds or thousands of PCM telemetry data frames, then sift through them repeatedly. These data can be converted to any number base and displayed, examined for any bit dropouts or changes (in particular, words or frames), graphically plotted, or statistically analyzed.

17. Key Words (Suggested by Author(s)) Decommutators Telemetry Data Compression
18. Distribution Statement

Unclassified - unlimited

STAR Category 32
19. Security Classif. (of this report)

20. Security Classif. (of this page) Unclassified
Unclassified
21. No. of Pages 8
22. Price $\mathrm{AO2}$ 